\title{
Roland Parenteau
}

Directeur, École nationale d'administration publique, Québec (ENAP)

(1970)

\section{“L’expérience de planification au Québec (1960-1969)”}

Un document produit en version numérique par Michel Fortin, bénévole,

Adjoint à la mairie, Ville de Saguenay, province de Québec

Courriel: micfortin@videotron.ca ou micfor@ville.chicoutimi.qc.ca

Dans le cadre de: "Les classiques des sciences sociales" Une bibliothèque numérique fondée et dirigée par Jean-Marie Tremblay, professeur de sociologie au Cégep de Chicoutimi Site web: http://classiques.uqac.ca/

Une collection développée en collaboration avec la Bibliothèque Paul-Émile-Boulet de l'Université du Québec à Chicoutimi Site web: http://bibliotheque.uqac.ca/ 
Cette édition électronique a été réalisée par Michel Fortin, bénévole, adjoint politique, Ville de Saguenay, à partir de :

\section{Roland Parenteau} bec)].

[Directeur de l'École nationale d'administration publique (Qué“L'expérience de la planification au Québec (1960-1969)”.

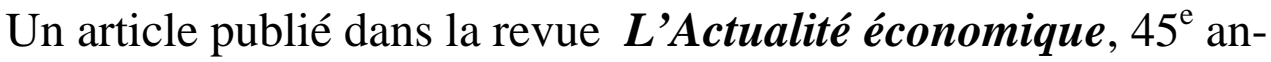
née, no 4, janvier-mars 1970, pp. 679-696.

Polices de caractères utilisée :

Pour le texte: Times New Roman, 14 points.

Pour les citations : Times New Roman, 12 points.

Pour les notes de bas de page : Times New Roman, 12 points.

Édition électronique réalisée avec le traitement de textes Microsoft Word 2004 pour Macintosh.

Mise en page sur papier format : LETTRE (US letter), 8.5’' x 11’')

Édition numérique réalisée le 26 février 2007 à Chicoutimi, Ville de Saguenay, province de Québec, Canada. 


\section{Roland Parenteau}

[Directeur de l’École nationale d'administration publique (Québec)].

\section{“L'expérience de la planification au Québec (1960-1969)”.}

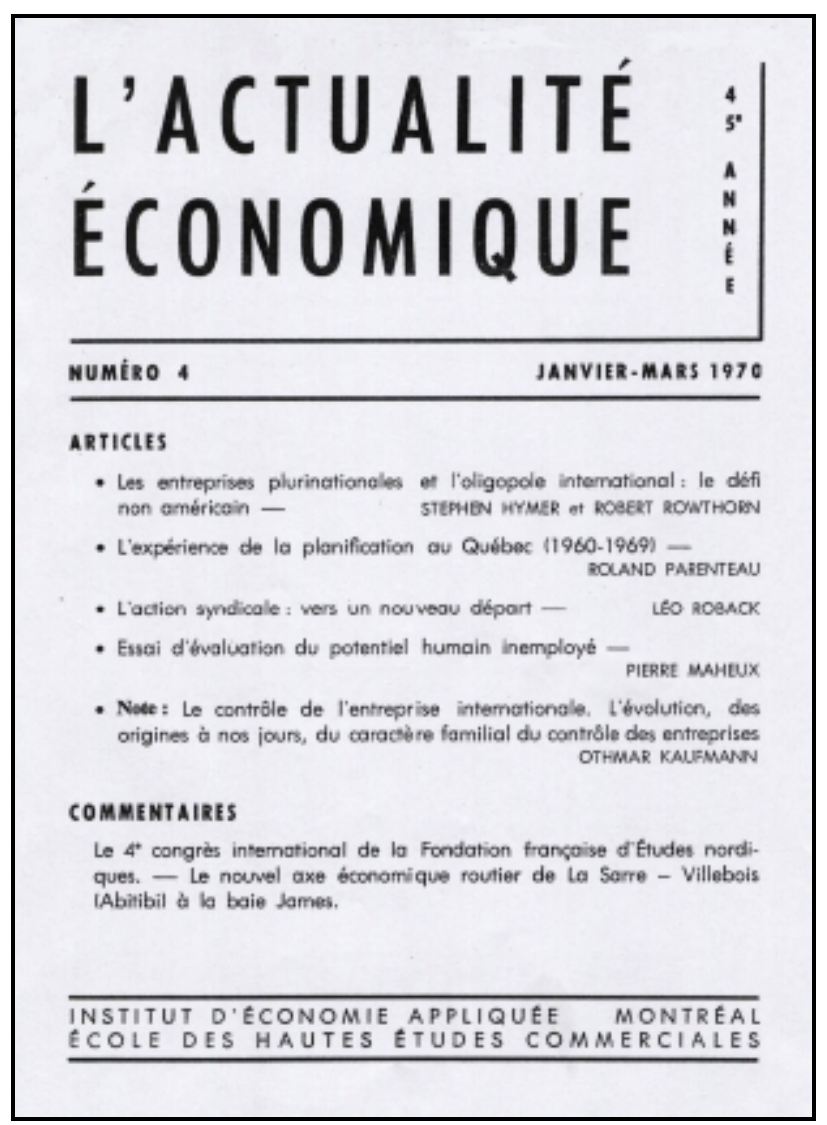

Un article publié dans la revue L'Actualité économique, $45^{\mathrm{e}}$ année, no 4, janvier-mars 1970, pp. 679-696. 


\section{Table des matières}

$\underline{\text { Introduction }}$

1. L’évolution des structures

1.1. Première période : février 1961 - septembre 1962

1.2. Deuxième période : septembre 1962 — avril 1964

1.3. Troisième période : avril 1964 — septembre 1966

1.4. Quatrième période : septembre 1966 — octobre 1968

2. Le concept de planification et le rôle de l'État

3. Conditions de la planification

3.1. Une connaissance trop imparfaite de la réalité

3.2. L'absence d'une volonté non équivoque, non seulement de planifier, mais d'en accepter les conséquences

3.3. L'absence d'accord sur les objectifs

3.4. La désuétude des structures d'exécution

3.5. Le partage des pouvoirs économiques entre deux niveaux de gouvernements

3.6. La perméabilité de l'économie québécoise aux influences étrangères 
Roland Parenteau (1921-)

[Directeur de l’École nationale d'administration publique (Québec)].

“L'expérience de la planification au Québec (1960-1969)”. 1

Un article publié dans la revue $L$ 'Actualité économique, 45e année, no 4, janvier-mars 1970, pp. 679-696.

\section{Introduction}

Les efforts en vue de planifier le développement socioéconomique du Québec, depuis 1960, se sont concrétisés par toute une série d'initiatives qui, même si elles ont donné naissance à certaines réalisations intéressantes, n'ont pas vraiment abouti aux résultats escomptés. En effet, on a assisté durant la période de 1960 à 1969 à la mise en place de toute une série de structures administratives nouvelles, au lancement de grandes entreprises économiques porteuses d'avenir, à un effort de pensée et de recherche d'une envergure qu'on n'avait jamais connue auparavant. Pourtant, toute cette agitation, sans avoir été totalement vaine, n'a pas conduit comme on aurait pu le souhaiter, à l'élaboration d'un véritable plan de développement pour le Québec.

C'est cette brève expérience de la planification que nous nous proposons de relater dans cet article, en nous appliquant non pas au détail historique, mais aux grandes lignes de l'évolution, en insistant particu-

1 Ce texte a d'abord été présenté sous forme de document de travail aux journées d'études de la Fédération des Travailleurs du Québec en novembre 1969. L’auteur l'a toutefois considérablement remanié. 
lièrement sur les contraintes d'ordre institutionnel et psychologique qui ont constitué autant de freins à la planification.

Notre propos est de centrer l'analyse sur le fonctionnement des pouvoirs publics et singulièrement sur celui du gouvernement du Québec, que nous considérons comme le promoteur de la planification, ce qu'il a été en fait, et comme le maître d'œuvre de la planification, ce qu'il aurait pu être. Nous estimons que la non-participation des autres agents économiques aux tentatives de planification qui se sont manifestées au cours de la période en question devenait fatale dès lors qu'on ne sentait pas une volonté incontestable des pouvoirs publics de s'engager dans cette voie.

Dans une première partie, nous rappellerons brièvement quelques événements qui ont marqué l'évolution des structures de planification, et plus particulièrement les activités du Conseil d'Orientation économique. Puis, nous décrirons comment la volonté de planifier les activités gouvernementales vient modifier le processus habituel de décision. Enfin, nous énumérerons quelques-unes des contraintes qu'on n'a pas réussi à surmonter totalement, ce qui explique le peu de succès obtenu.

\section{L'ÉVOLUTION DES STRUCTURES}

$\underline{\text { Retour à la table des matières }}$

Il faut se rappeler qu'en 1960, peu de gens savaient ce que signifiait la planification et quel rôle dans le développement économique un organisme de planification pouvait jouer. Néanmoins le parti libéral avait inscrit à son programme électoral de cette année-là la création d'un Conseil d'Orientation économique ${ }^{2}$. Effectivement, quelques

2 Il ne fait pas de doute que cette mention dans le programme du parti libéral avait été facilitée par la prise de position de la Chambre de Commerce de la province de Québec en 1958, en faveur de la planification. Ce n'est d'ailleurs pas pure coïncidence que le président de la Chambre cette année-là, Me René Paré, fut par la suite choisi comme premier président du Conseil d'Orientation économique. 
mois plus tard, soit en février 1961, ce Conseil était créé avec un double mandat :

- préparer un plan d'aménagement pour la province ;

- conseiller le gouvernement soit de sa propre initiative, soit sur demande, sur toute question d'ordre économique.

Même si la chose n'était pas exprimée très clairement dans la loi, il était entendu que le Conseil devait être le véritable organisme de planification du Québec et que, par conséquent, on devait lui accorder les moyens de remplir sa tâche, ce qui ne fut pas le cas. Bien plus, on vit au cours des années suivantes, apparaître toute une série dinitiatives de planification, en marge en quelque sorte du Conseil, ce qui n'était pas de nature à clarifier la situation. L'histoire du COEQ, depuis ses débuts en 1961 jusqu'à sa disparition en octobre 1968, se partage en quatre périodes.

\subsection{Première période : février 1961 - septembre 1962}

Cette période fut caractérisée par un certain nombre de recommandations faites par le Conseil au gouvernement et qui allaient déboucher sur la création ou le développement de quelques institutions économiques appelées à un avenir prometteur : Société générale de financement, complexe sidérurgique, regroupement des compagnies d'électricité, Régie des rentes, Caisse de dépôts et placements, etc. Faute de posséder à cette époque les éléments fondamentaux susceptibles d'être incorporés à un premier plan de développement, en l'absence d'une méthodologie de la planification, on a cru bon de mettre en place le plus rapidement possible, et cela sans présumer des objectifs éventuels d'un plan, certains instruments collectifs de développement qui ne pouvaient de toute façon donner leur pleine efficacité qu'après plusieurs années de rodage. 
Ce genre d'étude, suivi de recommandations, s'est poursuivi au cours des périodes subséquentes avec un succès moins évident mais non moins réel. Certaines réalisations du gouvernement, en effet, doivent être attribuées à l'activité déployée par le Conseil, même si on ne peut en établir la filiation d'une manière incontestable.

\subsection{Deuxième période : septembre 1962 - avril 1964}

$\underline{\text { Retour à la table des matières }}$

Délaissant quelque peu la technique du «travail à la pièce », le Conseil s'engagea résolument au cours de cette période dans la rédaction d'un plan global de développement dont la première phase devait être mise à exécution dès 1965.

Une activité intense fut déployée pendant plusieurs mois au sein de nombreux comités de planification à partir d'une ébauche de plan contenue dans un document élaboré par le Conseil et intitulé Document de base en vue de la planification. La méthodologie s'inspirait étroitement de l'expérience française, chacun des chapitres du plan devant être rédigé par un groupe de travail composé à la fois de fonctionnaires et de quelques spécialistes de l'extérieur.

On s'est vite rendu compte que ces efforts ne pouvaient mener très loin et qu'il était illusoire de parler de planification à ce stade. Le plan fut donc momentanément mis de côté au début de 1964 et le COEQ procéda à une évaluation de la situation qui l'amena à énumérer un certain nombre de conditions qu'il considérait comme autant de pré requis à une véritable planification. Ces conditions firent l'objet d'un rapport au gouvernement en septembre $1964{ }^{3}$. Celui-ci l'accepta en principe, mais en pratique les recommandations restèrent largement lettre morte.

3 Les exigences de la planification économique, rapport présenté par le Conseil d’Orientation économique, septembre 1964. 
Pour être juste, il convient toutefois de dire que l'activité intense déployée au sein des groupes de travail a provoqué un bouillonnement d'idées dont plusieurs ont réussi à faire leur chemin par la suite et à déboucher sur des initiatives gouvernementales, sans pour autant que le COEQ s'en vît toujours attribuer le crédit. Ainsi, certaines structures de planification commencèrent à fonctionner à cette époque, notamment le Conseil ministériel de la planification, composé de cinq ministres, le Comité permanent d'aménagement des ressources (CPAR) composé de sous-ministres. C'est au cours de la même période et avec la participation parfois déterminante du COEQ, que se développèrent les programmes ARDA, que fut mis en marche le BAEQ, que le Bureau de la Statistique du Québec se mit à construire son tableau interindustriel, etc.

\subsection{Troisième période : avril 1964 - septembre 1966}

$\underline{\text { Retour à la table des matières }}$

La mise en veilleuse du plan allait inciter le Conseil à se concentrer davantage sur la levée des hypothèses qui à son avis com $^{-}$ promettaient tout effort d'élaboration d'un plan digne de ce nom.

L'activité du COEQ s'est surtout manifestée dans des recherches de base (par exemple, étude du chômage saisonnier) et dans un effort en vue de sensibiliser l'appareil gouvernemental aux exigences de la planification. Une attention particulière a été apportée à l'étude des problèmes régionaux et à la création d'une conscience régionale de nature à faciliter la solution des problèmes d'aménagement du territoire (c'est l'époque de la création et du développement des conseils économiques régionaux). Cette période s'est terminée pour le COEQ par une longue réflexion sur son propre rôle et la préparation d'un mémoire qui équivalait à signer son propre arrêt de mort. Le COEQ, en effet, sensible aux difficultés de fonctionnement qui paralysaient son action, recommandait en septembre 1966, au nouveau gouvernement du Québec la création de deux organismes complémentaires : l'Office du plan et le Conseil de développement, destinés à le rempla- 
cer. Ainsi étaient nettement dissociées la fonction de planification et la fonction consultative.

Il faut ajouter que cette période fut fertile en initiatives gouvernementales de toutes sortes que l'on peut relier à la planification, sans que nécessairement le COEQ y ait été mêlé de près. C'est ainsi qu'on créa une foule de comités d'étude et de commissions d'enquête, que se développèrent dans plusieurs ministères des services de recherche ou de planification, qu'un projet de création d'un conseil provincial de la recherche et d'un centre de recherche industrielle (recommandés par le COEQ) se traduisit par la présentation à l'Assemblée nationale de deux bills.

\subsection{Quatrième période : septembre 1966 - octobre 1968}

$\underline{\text { Retour à la table des matières }}$

Cette période fut caractérisée par la longue attente de la réforme des structures de planification. Les recommandations du COEQ avaient été acceptées en principe par le Cabinet dès novembre 1966, mais ce n'est qu'en 1968 qu'elles furent traduites en législation.

Durant ce temps, le COEQ poursuivit un programme d'étude qui l'amena à concentrer son attention sur l'analyse approfondie des activités gouvernementales débouchant sur l'instauration d'un système de $\mathrm{PPB}$, des études sur l'armature urbaine et le phénomène de la déconcentration administrative. Il accordait, de plus, une attention particulière au problème de la participation de la population au processus de planification en analysant notamment le phénomène d'animation sociale ${ }^{4}$.

4 Cette réflexion donna lieu à une publication intitulée: L'animation, l'animation sociale, la consultation - essai de définition, Conseil d’Orientation économique du Québec, avril 1968. 
C'est aussi durant cette période que le COEQ, devenu dépositaire du plan du BAEQ, l'analysa en détail avec la collaboration des divers ministères concernés et prépara une recommandation qui servit ultérieurement de base à la signature d'une entente fédérale-provinciale. Il s'agissait d'une sorte de répétition générale de ce que pourrait être la préparation d'un plan global de développement avec la collaboration des ministères. En d'autres termes, le COEQ préparait les voies à un éventuel Office de planification.

En octobre 1968 fut donc effectivement créé cet Office de planification tant attendu. L'organisme nouveau pouvait se mettre résolument à la tâche et s'attaquer à l'élaboration d'un plan véritable. Il disposait à cet égard d'un ensemble de matériaux incomparablement supérieur à ceux dont disposait son prédécesseur, le COEQ en 1964. De plus, la loi de 1968 prévoyait que l'Office, organe gouvernemental chargé de la recherche et de la formulation des plans et politiques, serait flanqué de deux organismes consultatifs, une Commission interministérielle de planification groupant des hauts fonctionnaires et un Conseil de la planification, réunissant des représentants du secteur privé. Alors que la Commission interministérielle fonctionne déjà depuis février 1969, le gouvernement n'a pas encore jugé à propos de créer le Conseil.

Avant même que le nouvel Office de planification ait eu le temps de prendre son essor, une nouvelle modification fut apportée à la loi en 1969, laquelle élargissait considérablement les responsabilités de l'organisme qui prenait désormais le nom d'Office de planification et de développement.

Aux fonctions de planification déjà dévolues à l'Office, on ajoutait des responsabilités de coordination des plans et programmes et de réalisation de projets. C'est en vertu de ces amendements que l'administration ARDA - Québec, et l'ODEQ (Office de développement de l'est du Québec) furent intégrés à l'Office.

Ces modifications de structures, si prometteuses soient-elles à première vue, ne peuvent résoudre la difficulté essentielle rencontrée par toute tentative de planification à l'heure actuelle à savoir, d'une part, une certaine incompréhension quant à la nature même de la pla- 
nification du développement socioéconomique et, d'autre part, une absence d'efforts véritables en vue d'enlever les obstacles à la planification.

\section{LE CONCEPT DE PLANIFICATION ET LE ROLE DE L'ETAT}

$\underline{\text { Retour à la table des matières }}$

Il existe incontestablement beaucoup de confusion dans l'esprit des gens sur la nature de la planification. Une erreur très répandue consiste à assimiler la planification au dirigisme. Ainsi, on serait d'autant plus planificateur qu'on interviendrait davantage dans l'économie. En fait, ce que l'on néglige souvent de considérer, c'est la phase « élaboration du plan », pour ne s'attacher qu'à la phase "mise à exécution $»$.

Il est vrai que la planification est un attribut du pouvoir, mais celui-ci, dans un pays comme le nôtre, est partagé. Les pouvoirs publics, en tant que centre de décision et gardien de lintérêt général, jouent évidemment un rôle primordial dans l'élaboration d'un plan et dans sa mise à exécution. Notons bien qu'il s'agit ici de la planification du développement socioéconomique, car le terme de planification peut aussi être utilisé dans un sens plus restreint, par exemple la planification de l'éducation ou la planification des investissements publics.

On a coutume de considérer que la planification doit nécessairement déboucher sur la présentation d'un document appelé plan et qui sera plus ou moins détaillé, plus ou moins complet. Il ne s'agit là en réalité que d'une modalité. Essentiellement, la planification c'est un ensemble de techniques qui permettent aux divers agents économiques et singulièrement à l'État de prendre des décisions plus efficaces, plus rationnelles, plus cohérentes. Mais c'est aussi un état d'esprit, une attitude vis-à-vis les exigences de l'action. En un mot, la planification c'est un mode d'agir des responsables de l'intérêt public. Nous em- 
ployons à dessein ce dernier terme et non pas celui du gouvernement, car la planification du développement doit englober, comme on l'a vu, à tous les stades de son processus, tous les agents économiques significatifs, que ce soient les entreprises, les organisations syndicales ou les organismes publics et para-publics. Il est donc faux, dans un régime démocratique où les centres de décision sont multiples et dispersés, de croire que la planification peut être une responsabilité exclusive de l'État. Sans doute, celui-ci doit-il jouer le rôle d'initiateur et de maître d'œuvre dans l'élaboration du plan, mais au stade de l'exécution, il partage le pouvoir économique avec de nombreux autres agents dont la contribution plus ou moins empressée à la mise en oeuvre des directives du plan peut signifier le succès ou l'échec. Mais pour obtenir cette collaboration, il est évident que les intéressés doivent participer à la fois à la détermination des objectifs et au choix des moyens et des échéances. Dans une économie basée essentiellement sur la liberté économique et l'initiative privée, l'État ne peut donc imposer un plan de développement aux divers agents économiques.

Une courte réflexion sur le rôle de l'État dans l'économie moderne s'impose ici. L'activité des pouvoirs publics est multiforme : l'État est à la fois producteur de biens et de services, distributeur, consommateur et emprunteur ; il est aussi législateur, agent de contrôle, inspirateur et promoteur de politiques et de projets ; il peut être inventeur et agent de recherche; il est enfin employeur mais peut aussi servir d'arbitre.

L'ensemble de ces responsabilités peut se partager en deux groupes : celles qu'il assume à titre exclusif en tant que représentant la collectivité, comme attribut de sa souveraineté en quelque sorte, et celles qu'il assume en concurrence avec d'autres agents économiques. C'est ainsi que l'État comme employeur, comme acheteur, comme constructeur, comme emprunteur, doit partager des ressources limitées avec d'autres agents. Toutefois, son influence sur les marchés peut être déterminante, d'une part, à cause de la masse de ses opérations et, d'autre part, à cause de la possibilité qu'il a de se servir d'autres moyens que la négociation pour arriver à ses fins. Ainsi l'État, tout en acceptant en général les lois du marché, se permet parfois d'y 
déroger et de faire intervenir sa capacité législative ou un pouvoir de persuasion qui confine souvent à l'emploi de la force.

Cette ambivalence de rôles est inévitable dans le monde moderne. Elle a trouvé au cours des dernières années des applications nombreuses, en particulier dans le domaine de la négociation collective. On ne sait plus à certains moments si l'État joue le rôle d'employeur ou de législateur ou d'arbitre dans sa propre cause.

À l'égard de l'achat de certaines fournitures et de services, l'État possède parfois une situation de monopole d'achat qui lui permet de dicter ses conditions aux fournisseurs. De même dans le domaine financier, les emprunts des pouvoirs publics sont d'une importance telle qu'ils sont déterminants sur les taux d'intérêt. En revanche, l'État est en mesure d'exercer une influence sur l'offre de monnaie par toutes mesures fiscales ou autres, contribuant à encourager l'épargne et ainsi à augmenter le réservoir dans lequel il pourra lui-même puiser.

Ces considérations générales sont nécessaires pour comprendre le rôle de l'État dans le développement économique. En fait, les gouvernements modernes possèdent une puissance en matière économique que la plupart des dirigeants politiques ne soupçonnent même pas, habitués qu'ils sont à assimiler pouvoir économique à propriété des moyens de production. Cette notion capitaliste du pouvoir a déjà été rejetée depuis longtemps dans le monde de l'entreprise privée, puisqu'on sait que ce ne sont pas en général les propriétaires qui prennent les décisions.

Compte tenu de ce double rôle de l'État, quels changements apporte la planification dans le processus de décision ? Nous serions tenté de schématiser le processus décisionnel de la façon suivante, en distinguant quatre étapes.

La première étape est celle de la sensibilisation à un problème ou à une situation. L'initiative sur ce plan peut être de diverse nature. Il peut s'agir de l'expression d'un besoin généralement reconnu, de pressions particulières venant de groupes d'intérêt, d'expériences étrangères, des résultats d'une recherche approfondie. De toute façon l'identification du problème ainsi que certains éléments de solution sont 
transmis aux pouvoirs publics qui les étudient, procèdent à des consultations plus ou moins étendues et en arrivent à une proposition qui est transmise au centre de décision lui-même, soit le conseil des ministres. C'est le stade de l'élaboration. Certains projets s'arrêtent là puisqu'ils sont refusés. D'autres franchissent les étapes ultérieures, soit d'abord celle de la décision se traduisant par l'utilisation de l'une ou de l'autre des modalités de l'action gouvernementale : nouvelle législation, modification de lois ou de règlements antérieurs, création de nouvelles institutions ou de nouveaux services, mise au point de projets spécifiques, se répercutant dans les budgets déterminés. Enfin, l'exécution intervient selon les conditions fixées au stade de la décision.

Ce modèle de décision peut être utilisé dans un contexte planifié ou non planifié (voir diagrammes, pages 8 et 9). Il faut se rappeler à cet égard quiun gouvernement n'est pas un organisme monolithique comportant une seule ligne d'autorité représentée par la pyramide traditionnelle. C'est, au contraire, un organisme complexe où les responsabilités sont partagées d'une façon permanente entre administrations parallèles.

On s'attend qu'il existe une certaine cohérence dans les décisions gouvernementales pour peu qu'il s'agisse de sujets portés à la connaissance de l'organisme central de décision, à savoir le conseil des ministres. Mais on oublie souvent que beaucoup de décisions administratives, voire même des politiques générales, sont élaborées et mises à exécution par les ministères, régies, commissions, offices, etc., chacun jouissant d'une économie plus ou moins complète dans le cadre des pouvoirs généraux qui lui sont confiés par la loi. Il n'est donc pas étonnant que dans un contexte non planifié les objectifs poursuivis soient souvent incompatibles entre eux ou même parfois inexistants et les mesures adoptées inefficaces sinon contradictoires. En pratique, le conseil des ministres peut difficilement jouer son rôle unificateur et empêcher le chevauche-ment des juridictions à cause de l'énormité de l'appareil et l'absence de mécanisme d'établissement de priorités d'action.

Dans un contexte planifié, lequel suppose la plupart du temps la création d'un organisme central chargé non seulement d'élaborer le 
plan global de développement, mais d'en surveiller l'exécution, le processus de décision est un peu plus complexe. Sans doute, l'élaboration initiale des politiques peut encore relever d'instances décentralisées, mais en collaboration avec l'organisme de planification qui vérifie leur cohérence par rapport à des objectifs de plus grande envergure qu'il a contribué à faire accepter par l'État pour l'ensemble de la société. Les décisions du gouvernement se trouvent donc ainsi mieux éclairées parce qu'elles tiennent compte de toute la complexité des facteurs en cause, tels qu'exposés dans le guide général de politique qu'est le plan.

En pratique, le cheminement des projets est le même, mais on y ajoute, à certains moments stratégiques, lintervention directe ou indirecte diun organisme central : notamment au stade qui précède immédiatement la prise de décision par le conseil des ministres, puis au stade de l'exécution des programmes où le besoin de coordination s'impose si l'on veut obtenir le maximum d'impact.

Il est important de rappeler ici que si la planification s'exprime souvent par la publication d'un document complexe exposant les objectifs et les solutions proposés avec force graphiques et statistiques, cette formule ne constitue pas la seule modalité à envisager. La planification étant un processus continu, on peut fort bien concevoir une situation dans laquelle les objectifs ne seraient pas exprimés aussi clairement et aussi globalement. En d'autres termes, on peut planifier par une série d'approches successives en commençant par utiliser des moyens assez grossiers, mais qui sont appelés à se raffiner sans cesse davantage. L'important c'est qu'un organisme central ne perde pas de vue l'ensemble du processus. 


\section{Le processus de décision gouvernemental}

1. Contexte non planifié

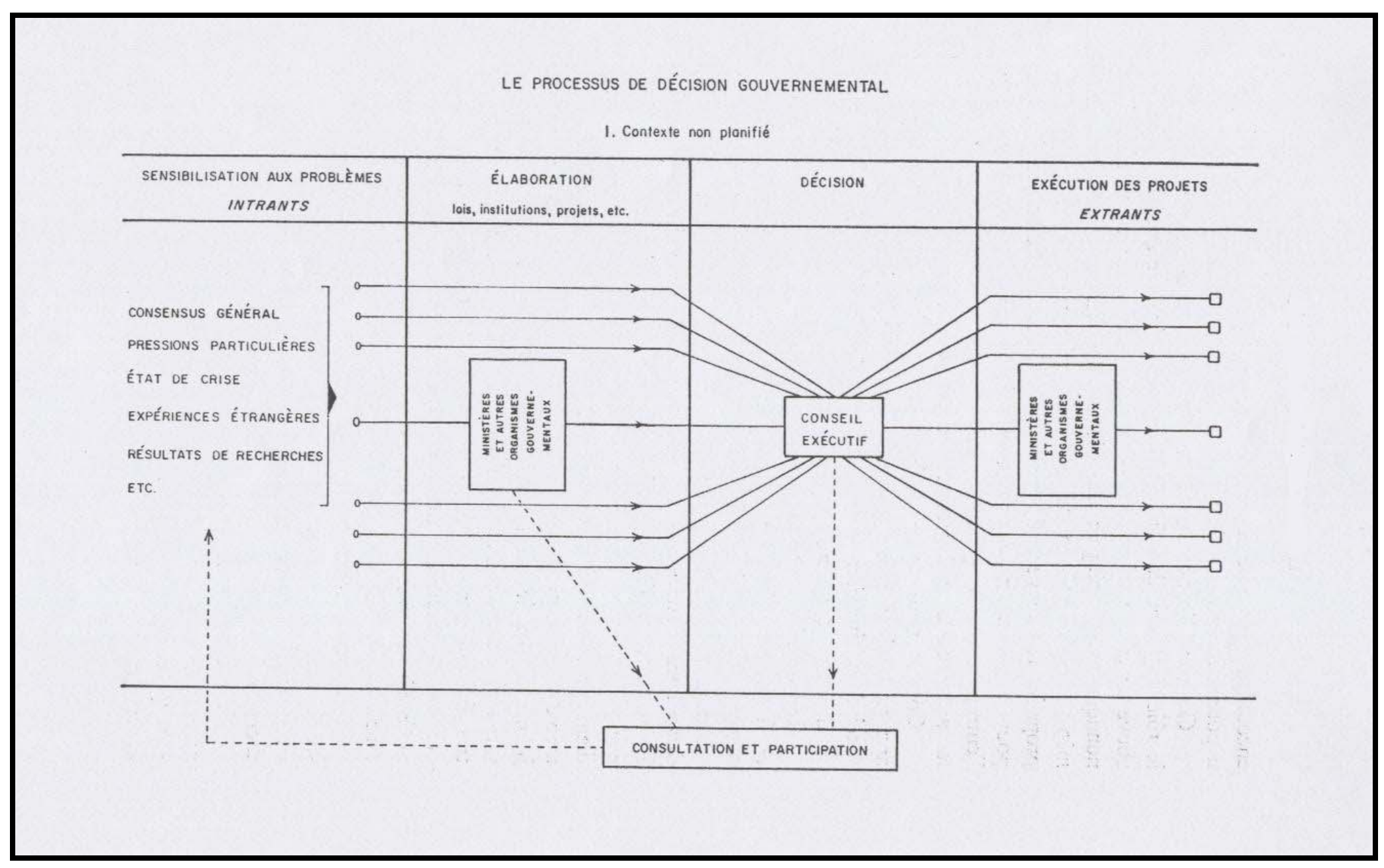




\section{Le processus de décision gouvernemental \\ 2. Contexte planifié}

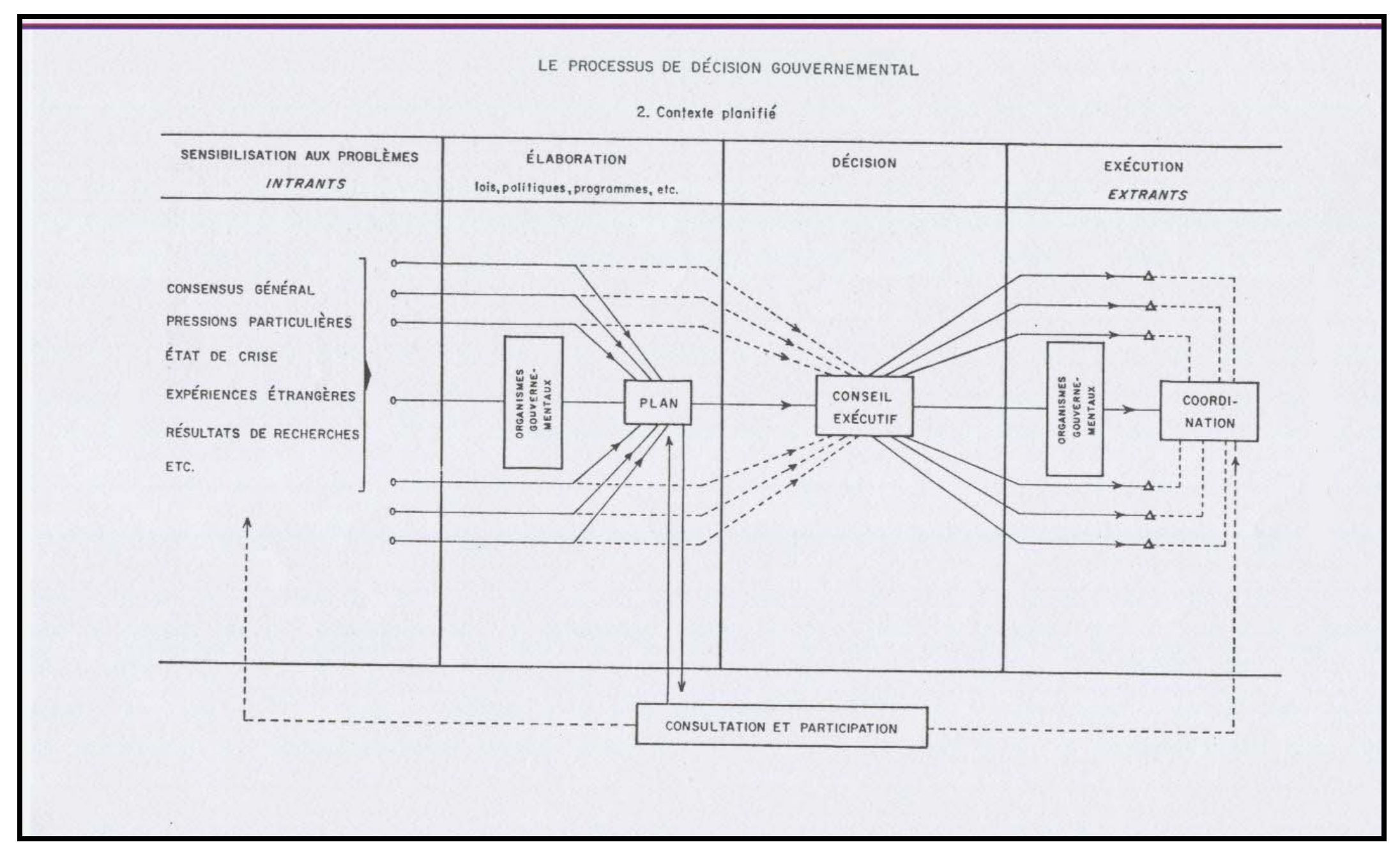




\section{CONDITIONS DE LA PLANIFICATION}

$\underline{\text { Retour à la table des matières }}$

Quelle que soit la conception qu'on se fait de la planification, il faut se rappeler que l'activité économique est exercée par de nombreux agents plus ou moins regroupés et dont les intérêts sont parfois opposés. Les décisions significatives influençant par exemple l'épargne ou les importations peuvent découler d'une stratégie syndicale tout autant que de gestes posés par des chefs d'entreprise ou une réglementation émanant de l'État. Il ne suffit donc pas de confier à celuici le rôle de planificateur et de s'en laver les mains par la suite. La planification dans tout pays démocratique ne peut qu'être une oeuvre collective. Et ce n'est pas uniquement par attachement pour la démocratie qu'il faut affirmer cela, mais par simple nécessité. Le gouvernement ne peut pas fixer des priorités que n'accepterait pas la majorité des agents économiques. Et ceux-ci ont beaucoup de moyens à leur disposition de compromettre la réalisation d'un plan, quel qu'il soit.

Cela étant dit, la responsabilité de l'État en matière de planification est double : il a le devoir de planifier sa propre activité, de coordonner les programmes des divers organes qui émanent de lui, mais il a une responsabilité plus large, celle de servir de maître d'œuvre dans l'élaboration d'un plan global de développement.

Une fois posé ce principe, que dans le cadre de cet article nous appliquons au Québec, demandons-nous pourquoi en dépit d'efforts louables déployés depuis près d'une dizaine d'années, nous ne soyons pas encore parvenus à élaborer un plan formel de développement.

Les obstacles à la planification sont nombreux et nous ne voudrions en énumérer que quelques-uns. Il est peut-être important de dire tout de suite que, contrairement à ce qu'on est souvent porté à penser, ces obstacles ne tiennent pas la plupart du temps au comportement des individus, à leur plus ou moins bonne volonté, à leur 
égoïsme ou à leur soif du pouvoir. Bien plus, ils n'ont que peu de rapports avec les diverses classes sociales ou les groupes d'intérêt. On ne peut affirmer, par exemple, que les hommes daffaires sont opposés à la planification alors que les ouvriers y sont favorables. L'inverse n'est pas vrai non plus.

Le problème est social et institutionnel : il relève, en d'autres termes, des attitudes collectives qui sont elles-mêmes le résultat d'une longue tradition de libéralisme économique. Il relève aussi d'un cadre institutionnel qui, bien que complètement dépassé, n'a pas subi de changement majeur depuis plus d'un siècle. L'expérience québécoise de planification s'est heurtée, entre autres, aux difficultés suivantes.

\subsection{Une connaissance trop imparfaite de la réalité}

\section{$\underline{\text { Retour à la table des matières }}$}

Cette affirmation ne remporte pas l'adhésion de tous. Beaucoup de gens, en effet, estiment que l'on a fait suffisamment d'études et que le temps de l'action est arrivé. Il nen reste pas moins vrai qu'on sait très peu de choses sur les mécanismes du développement économique, sur les facteurs de localisation des activités, sur l'évolution des comportements des agents économiques. Le résultat c'est que de nombreuses décisions destinées soi-disant à corriger certains maux économiques ou à stimuler la croissance sont autant de coups d'épée dans l'eau. En fait, dans le passé on n'a pas accordé suffisamment d'importance à la recherche et à la réflexion, pressé qu'on a été d'agir sous la pression des clameurs qui venaient de toute part. 


\subsection{L'absence d'une volonté non équivoque, non seulement de planifier, mais d'en accepter les conséquences,}

à savoir une certaine perte d'autonomie des groupes au profit d'une concertation pour l'ensemble de la société. Cet aspect de la planification est souvent mésestimé. L'individualisme qui caractérise encore fortement notre régime économique incite les divers agents à se ménager des positions de force qui leur permettent de négocier par la suite en vue d'obtenir le maximum d'avantages. Or, une société dans laquelle les mécanismes d'ajustement des salaires, des prix, des taux d'intérêt sont basés principalement sur des rapports de force, est fort éloignée de la planification, laquelle se fonde plutôt sur la concertation, sans pour cela ignorer l'existence d'intérêts divergents.

\subsection{L'absence d'accord sur les objectifs}

Même si la réalité nous était parfaitement connue et si on désirait vraiment planifier le développement, cela ne signifierait pas pour autant qu'il serait facile de fixer des objectifs communs. Évidemment, à un certain niveau d'abstraction, on peut facilement énumérer une liste d'objectifs sur lesquels on peut réaliser une entente. Ainsi, à peu près tout le monde voudrait :

- qu'on construise des logements à loyer modique ;

- que les prix des denrées n'augmentent pas ;

- que les taxes restent les mêmes et si possible, soient réduites ;

- que la semaine de travail soit raccourcie et les vacances allongées ;

- que les taux des prestations sociales soient augmentés ;

- que l'enseignement à tous les niveaux soit gratuit ;

- que tout le monde ait du travail et à des taux rémunérateurs ;

- qu'on améliore le réseau de voirie ;

- que le salaire minimum soit haussé ; 
- que nos villes soient moins laides, etc.

Le problème, cest que les ressources dont nous disposons étant limitées, en d'autres termes, dans l'impossibilité où nous nous trouvons de réaliser ces objectifs pleinement et en même temps, il faut faire un choix. Et c'est là que les désaccords apparaissent. Les uns voudraient privilégier le développement des régions excentriques, les autres voudraient que les prix soient plafonnés ou encore que les hausses de salaires soient freinées. Les uns établissent comme priorité la construction de logements bon marché, les autres voudraient des villes plus belles et plus fonctionnelles, les uns souhaitent la gratuité scolaire immédiate et complète alors que d'autres voudraient supprimer le chômage grâce à d'immenses travaux publics.

Le gouvernement lui-même est soumis à des pressions, parfois contradictoires et toujours concurrentes au point de vue de l'utilisation des ressources. Son hésitation perpétuelle vient de ce que les hommes politiques eux-mêmes n'ont pas la même perception des priorités. Le résultat net de ce jeu de puissance et en l'absence d'un guide général sur lequel on se serait entendu au préalable, c'est qu'on essaie en pratique de contenter tout le monde en accordant successivement des avantages à telle ou telle catégorie d'individus, en cédant sur tel ou tel point. L'État se trouve dans une position défensive continuelle de telle sorte que tout le monde a l'impression que ses aspirations ou ses demandes sont refusées alors que les budgets s'accroissent tout de même à un rythme accéléré. On assiste à une frustration collective et c'est l'État qui porte seul l'odieux des refus. Ce serait précisément le rôle d'un plan, non pas nécessairement de mettre tout le monde d'accord, mais de soumettre à une majorité le choix d'objectifs clairs et précis.

\subsection{La désuétude des structures d'exécution}

\section{$\underline{\text { Retour à la table des matières }}$}

Nos institutions politiques et administratives sont peut-être ce qu'il y a de plus démodé dans notre société. Elles ont été conçues pour un contexte tout à fait différent dans lequel l'État jouait surtout le rôle d'agent de police et de gestionnaire du domaine public. D'où ce par- 
tage de responsabilités entre de nombreux ministères, chacun très jaloux de son autonomie. Cette dernière est d'ailleurs accentuée par le mode traditionnel de recrutement des fonctionnaires selon lequel une profession donnée domine à toutes fins pratiques un ministère spécifique. La situation est aggravée par l'habitude qu'on a prise de créer, pour des fins diverses, toute une série d'offices, de commissions et de régies dont l'esprit de collaboration, c'est le moins qu'on puisse dire, n'est pas des plus empressés.

Pourtant, tout le monde déplore la situation actuelle mais personne ne sait par quel bout commencer. Pour être juste il faut mentionner que certains progrès ont été réalisés depuis quelques années en matière de coordination interministérielle, mais la tâche à accomplir reste immense. De plus, ce sont les institutions qui sont en cause plus que la bonne volonté des individus. En réalité, c'est à une réforme totale et en profondeur du système administratif actuel qu'il faut procéder.

\subsection{Le partage des pouvoirs économiques entre deux niveaux de gouvernements}

$\underline{\text { Retour à la table des matières }}$

Voilà un obstacle de taille dont, en général, on ne mesure pas suffisamment l'importance. Les pouvoirs économiques sont partagés par la constitution entre deux pouvoirs souverains, de sorte qu'une conjugaison d'efforts se révèle vraiment ardue. Il faut ajouter que dans le passé le gouvernement fédéral a adopté une attitude de grand frère qui l'amenait à élaborer seul les politiques et à placer les provinces devant un fait accompli. Or, à moins d'une révision radicale de cette attitude, la planification restera toujours difficile dans un contexte fédératif. Il ne suffit donc pas à cet égard que les deux gouvernements soient prêts à collaborer au stade de l'exécution, ce qui est souvent le cas, mais il faudrait en arriver à l'élaboration en commun des politiques et des programmes, dans tous les domaines impliquant des actions parallèles. Il semble qu'on soit très loin de ce mode de coopération, pour des raisons qu'il serait trop long d'énumérer ici. 


\subsection{La perméabilité de l'économie québécoise aux influences étrangères}

$\underline{\text { Retour à la table des matières }}$

Cette influence s'exerce de multiples façons dont les unes très connues (exemple : capitaux étrangers, dépendance de marchés extérieurs, etc.) et les autres plus subtiles, comme par exemple, l'assujettissement de plus en plus généralisé à l'égard des techniques et des comportements américains. Cette situation n'a pas que des inconvénients, bien au contraire, mais elle n'en limite pas moins la marge de manœuvre laissée aux pouvoirs publics pour des politiques authentiquement québécoises.

L'énumération de ces contraintes n'a pas pour but, malgré son apparent caractère pessimiste, de démontrer que la planification du développement socioéconomique est impossible au Québec. Elle vise à mieux identifier ces contraintes pour que la collectivité puisse les supprimer, dans la mesure évidemment où l'on tient à ce que le développement socioéconomique ne soit pas uniquement le résultat du hasard ou des forces économiques laissées à elles-mêmes.

Voilà donc quelques-unes des considérations qu'une observation attentive de l'évolution récente du Québec est de nature à susciter. Elles soulèvent une question encore plus fondamentale, à savoir est-il vraiment possible de planifier le développement socioéconomique dans une économie encore largement influencée par le libéralisme économique et aux prises dans une très large mesure avec les lois du marché ? Notre réponse est affirmative. L'expérience de certains pays d'Europe, notamment de la France, révèle que la "performance » de l'économie peut être sensiblement améliorée par la planification pourvu qu'on ne prétende pas faire jouer à celle-ci un rôle qu'elle est incapable de jouer. Vouloir, par exemple, une réalisation quasi automatique des objectifs quantitatifs qu'on s'est fixés est un leurre. On peut même affirmer qu'on n'y arrive pas davantage dans les pays à direction unifiée, comme les pays socialistes. L'important c'est de se demander si l'intervention d'un processus de planification ne permet pas de réali- 
ser un taux de croissance plus élevé, d'éviter plus de gaspillage de ressources que dans le cas contraire.

À cet égard, tout le monde admet que des progrès sensibles non seulement s'imposent dans notre pays, mais pourraient être obtenus sans grand effort d'imagination. Encore faut-il qu'on réussisse à mobiliser les divers agents économiques en vue de cet objectif. L'expérience récente du Québec à cet égard, si elle ne sest pas soldée par une réussite incontestable a eu au moins le mérite de montrer les divers obstacles qu'on rencontrera fatalement et d'en évaluer le poids relatif.

Roland Parenteau

Directeur de l'École nationale d’administration publique (Québec)

Fin du texte 